\title{
High power conditioning and benchmarking of planar nitrogen-incorporated ultrananocrystalline diamond field emission electron source
}

\author{
Jiahang Shao , $^{1, *}$ Mitchell Schneider $\odot,{ }^{2, \star}$ Gongxiaohui Chen $\odot,{ }^{3, \$}$ Tanvi Nikhar $\odot{ }^{2}$ \\ Kiran Kumar Kovi, ${ }^{4}$ Linda Spentzouris $\odot,{ }^{3}$ Eric Wisniewski, ${ }^{1}$ John Power, ${ }^{1}$ \\ Manoel Conde, ${ }^{1}$ Wanming Liu, ${ }^{1}$ and Sergey V. Baryshev $\circledast^{2, \dagger}$ \\ ${ }^{1}$ Argonne National Laboratory, Lemont, Illinois 60439, USA \\ ${ }^{2}$ Michigan State University, East Lansing, Michigan 48824, USA \\ ${ }^{3}$ Illinois Institute of Technology, Chicago, Illinois 60616, USA \\ ${ }^{4}$ Euclid Techlabs LLC, Bolingbrook, Illinois 60440, USA
}

(Received 30 July 2019; published 11 December 2019)

\begin{abstract}
Planar nitrogen-incorporated ultrananocrystalline diamond $[(\mathrm{N}) \mathrm{UNCD}]$ has emerged as a unique field emission source attractive for accelerator applications because of its capability to generate a high charge beam and handle moderate vacuum conditions. Most importantly, (N)UNCD sources are simple to produce: Conventional high aspect ratio isolated emitters are not required to be formed on the surface, and the actual emitter surface roughness is on the order of only 10-100 $\mathrm{nm}$. A careful reliability assessment of $(\mathrm{N}) \mathrm{UNCD}$ is required before it may find routine application in accelerator systems. In the present study using an L-band normal conducting single-cell rf gun, a (N)UNCD cathode has been conditioned to $\sim 42 \mathrm{MV} / \mathrm{m}$ in a well-controlled manner. It reached a maximum output charge of $15 \mathrm{nC}$ corresponding to an average current of $6 \mathrm{~mA}$ during an emission period of $2.5 \mu \mathrm{s}$. Imaging of the emission current revealed a large number of isolated emitters (density over $100 / \mathrm{cm}^{2}$ ) distributed on the cathode, which is consistent with previous tests in dc environments. The performance metrics, the emission imaging, and the systematic study of emission properties during rf conditioning in a wide gradient range assert (N)UNCD as an enabling electron source for $\mathrm{rf}$ injector designs serving industrial and scientific applications. These studies also improve the fundamental knowledge of the practical conditioning procedure via a better understanding of the emission mechanisms.
\end{abstract}

DOI: 10.1103/PhysRevAccelBeams.22.123402

\section{INTRODUCTION}

The field emission cathode (FEC) is a viable choice for $\mathrm{rf}$ injectors in many industrial and scientific applications [1-10]. It has several clear advantages compared to thermionic cathodes and photocathodes. First, it is simple, as no heating system or laser is required to facilitate electron emission $[6,7,9]$. Second, it has the capability to deliver a high output current with maximum current densities on the order of $10^{8} \mathrm{~A} / \mathrm{cm}^{2}$ [5]. Third, it has the potential to reach ultralow emittance and energy spread via various gating methods $[4,6,11,12]$. The way is being paved toward the realization of high-performance FEC-based injectors.

\footnotetext{
*jshao@anl.gov

serbar@msu.edu

M. S. and G. C. contributed equally to this work.
}

Published by the American Physical Society under the terms of the Creative Commons Attribution 4.0 International license. Further distribution of this work must maintain attribution to the author(s) and the published article's title, journal citation, and DOI.
Maintaining high FEC performance over long-term operation remains an important goal. Various advanced field emitter materials and configurations are currently under intense investigation in $\mathrm{dc}$ and $\mathrm{rf}$ environments. For high power vacuum systems, materials of interest are often carbon-derived substances such as the carbon nanotube $[9,13,14]$, nanocrystalline diamond (NCD) [15-17], nitrogen-incorporated ultrananocrystalline diamond $[(\mathrm{N})$ UNCD] [6,8,18], and others [19-21]. For aggressive high oxygen content environments like thrusters, diamond and boron nitride are under intense study [22]. Configurations of interest include Spindt-type high aspect ratio single emitters or emitter arrays and planar nanometer-roughness emitters.

Planar (N)UNCD is a promising material that yields a high current and allows for simplicity and scalability in fabrication. Although the exact mechanism of field emission from diamond surfaces is still under debate, previous experiments have shown that emission originates from the $s p^{2}$ grain boundaries [23,24]. The high grain boundary density of (N)UNCD material could therefore potentially 
lead to a high current density. To date, planar (N)UNCD cathodes have been tested and shown to perform in normal conducting rf guns $(20-70 \mathrm{MV} / \mathrm{m})[6,8]$, in a superconducting of gun (1 MV at 2-4 K) [25], and in de setups $(1-20 \mathrm{MV} / \mathrm{m})$ [18]. The full operational range, from turnon up to the breakdown field, must be explored systematically before (N)UNCD can be considered for accelerator applications. These may include industrial systems (surface fields $1-20 \mathrm{MV} / \mathrm{m}$ ) or scientific systems (surface fields up to $100 \mathrm{MV} / \mathrm{m}$ ). Addressing the fundamental emission mechanism puzzle is also critical for successful (N)UNCD deployment.

The study presented here extends planar (N)UNCD cathode high pulsed power characterization during if conditioning. Detailed emission properties were recorded as the macroscopic $E$ field was conditioned from $\sim 8$ to $\sim 42 \mathrm{MV} / \mathrm{m}$; these included the current and current density, effective emission area $A_{e}$, field enhancement factor $\beta$ and microscopic electric field, turn-on field, and temporal emission stability. The metrics evolution of the emission parameters was analyzed and interpreted in the framework of a unique density-of-states structure of (N)UNCD [26]. High-resolution imaging of the (N)UNCD emitting surface in the rf gun environment showed a large number of localized emitters (density over $100 / \mathrm{cm}^{2}$ ) distributed across the surface. This result is consistent with results obtained in dc setups $[18,27,28]$.

This paper is organized as follows: Sec. II presents the cathode preparation; Sec. III introduces the experimental setup; Sec. IV provides a detailed description of the experimental methods, procedures, and results; Sec. V discusses the experimental results and presents a hypothesis for the emission evolution mechanism during rf conditioning; and Sec. VI summarizes the study and outlines future work.

\section{CATHODE PREPARATION}

\section{A. Cathode assembly}

The cathode plug ( $34.7 \mathrm{~mm}$ tall and $20 \mathrm{~mm}$ in diameter) is designed as a three-part assembly with an aluminum body, an aluminum middle piece, and a stainless steel top piece, as illustrated in Fig. 1. The design meets the installation requirements of the L-band photocathode rf gun test stand $[8,12,29,30]$ and enables convenient material synthesis onto the thin top part. The parts are aligned with each other and assembled together using internal vented screws. The electrical contact between the cathode assembly and the rf gun is ensured by a spring around the cathode body.

\section{B. Cathode synthesis}

The synthesis procedure of depositing the (N)UNCD material onto the top piece is described as follows. (i) The top piece was polished to 100 -nm-level roughness to avoid any high aspect ratio site-induced emission. The stylus profilometer measurement and the spatial Fourier

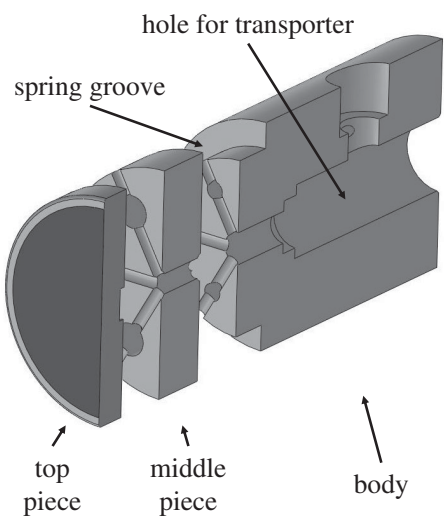

FIG. 1. Cutaway drawing of the cathode assembly. The dark part of the top piece represents the (N)UNCD material. The vented screws holding the parts together are not shown.

analysis [31,32] suggest the maximum physical field enhancement factor is lower than 1.01, as illustrated in Fig. 2. (ii) A molybdenum buffer layer of $150 \mathrm{~nm}$ thickness was deposited using magnetron sputtering. (iii) The top piece underwent an ultrasonic seeding procedure that made use of diamond slurry (Adamas Technologies) with a particle size of 5-10 nm. (iv) The (N)UNCD material was deposited
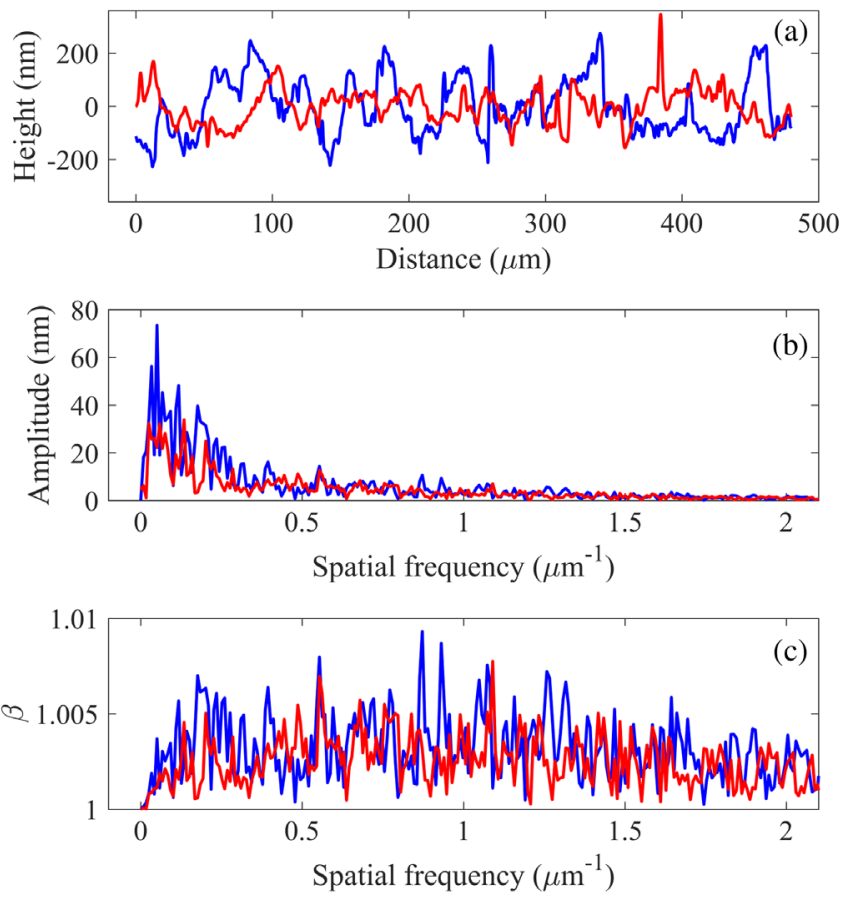

FIG. 2. Surface roughness analysis of the polished top piece before (blue lines) and after (red lines) the high power test. The two measurements were conducted at two close sites within $1 \mathrm{~mm}$ distance. (a) Surface profile. Before the high power test, the peakto-peak and root-mean-square roughness is 503 and $108 \mathrm{~nm}$, respectively. After the high power test, the peak-to-peak and rootmean-square roughness is 503 and $66 \mathrm{~nm}$, respectively. (b) Spatial frequency spectrum. (c) Field enhancement factor. 


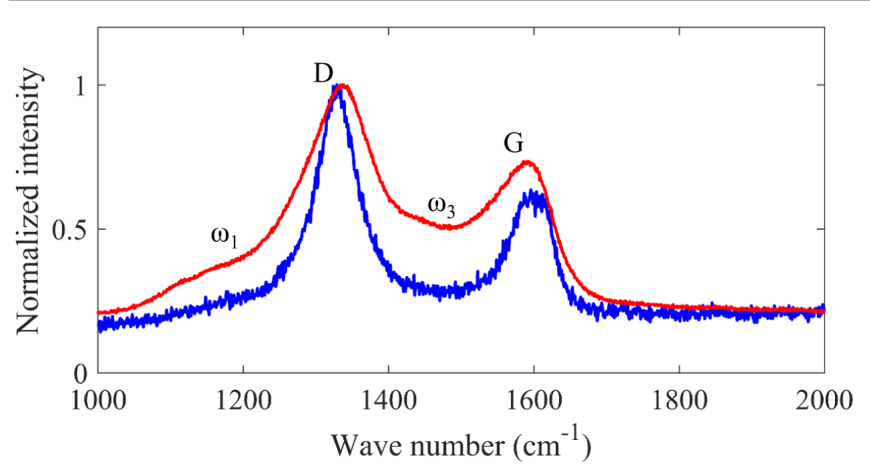

FIG. 3. Raman spectra taken at different locations across the (N)UNCD surface showing carbon phase variation. Measurements were performed using a primary probe wavelength of $633 \mathrm{~nm}$. Red curve: Location with nearly canonical (N)UNCD spectra containing D and G bands intrinsic to UNCD and $\omega_{1}$ and $\omega_{3}$ bands showing the presence of hydrocarbon transpolyacetylene chains in grain boundaries. Blue curve: Location demonstrating stronger graphitization of UNCD in which the right-hand shift of the $\mathrm{G}$ band suggests that grain boundaries are better crystallized due to a higher local synthesis temperature signified by the absence of $\omega_{1}$ and $\omega_{3}$ bands of t-PA that decomposes above $1100 \mathrm{~K}$.

using microwave-assisted plasma chemical vapor deposition (Lambda Technologies Inc.) operated at 915 MHz. During growth, the total pressure was 56 Torr, the microwave power was $2.3 \mathrm{~kW}$, and individual gas flows for $\mathrm{Ar}, \mathrm{CH}_{4}$, and $\mathrm{N}_{2}$ were 3,160 , and $40 \mathrm{sccm}$, respectively.

During the growth of (N)UNCD via chemical vapor deposition, there are competing processes that form the diamond $s p^{3}$ phase and the graphitic $s p^{2}$ phase. These should be in the right proportion to allow mechanical strength and adhesion and to obtain high conductivity and emission efficiency. Here, high emission efficiency means a low turn-on field $(1-10 \mathrm{MV} / \mathrm{m})$ and a high field enhancement factor (100-1000). Given that grain boundaries $\left(s p^{2}\right.$ phase) facilitate emission, the (N)UNCD efficiency can be tuned through the $s p^{3}$-to-s $s p^{2}$ ratio $[16,33]$. Compared to our previous growth protocol executed at $850^{\circ} \mathrm{C}$ [8], the synthesis temperature was increased by $50^{\circ} \mathrm{C}$ to induce a larger fraction of ordered graphitic $s p^{2}$ phase [16].

Raman spectroscopy revealed that the graphitic phase increased (as compared to Ref. [8]) but at the same time appeared to nonuniformly distribute across the surface, as illustrated in Fig. 3. Some locations (red curve) demonstrated nearly canonical (N)UNCD spectra. Meanwhile, other locations (blue curve) showed stronger graphitization due to a higher local temperature which is evidenced by the disappearance of $\omega_{1}$ and $\omega_{3}$ bands associated with transpolyacetylene (t-PA) [34]. All characterized locations demonstrate the strong $\mathrm{D}$ band representing the diamond grain $s p^{3}$ host matrix and the $\mathrm{G}$ band that is associated with the graphitic $s p^{2}$ grain boundaries. Locations such as that represented by the blue curve in Fig. 3 are expected to have grain boundaries that are better crystallized compared to locations such as that represented by the red curve.

In spray-deposited films comprised of diamond and graphite powders mixed at varied diamond-to-graphite ratios, that are model systems with predesigned nonuniform diamond-to-graphite distribution, electron emission was found to be nonuniform across the surface [27]. Therefore, it could be speculated that such a nonuniform diamond-tographite $\left(s p^{3}\right.$-to-s $\left.s p^{2}\right)$ content distribution is responsible for the nonuniform emission across the nanodiamond surface. This was also observed in previous dc studies [18,28], and in both cases it was shown that surface topography cannot explain the enhanced field emission at a low turn-on field.

The average work function of the (N)UNCD surface after deposition was measured to be $4.0 \mathrm{eV}$ by a Kelvin probe, close to the ones reported in previous studies $[35,36]$.

\section{EXPERIMENTAL SETUP}

The experiment was conducted using the Argonne Cathode Test-stand (ACT) beam line at the Argonne Wakefield Accelerator (AWA) facility $[8,12,29,30]$, which is illustrated in Fig. 4. The ACT beam line is equipped with a single-cell normal conducting photocathode rf gun operated in the L band at $1.3 \mathrm{GHz}$. The beam line currently runs at a $2 \mathrm{~Hz}$ repetition rate with a full width half maximum (FWHM) pulse length of $6 \mu \mathrm{s}$. Cathodes with various shapes and materials can be tested thanks to the detachable cathode design. A frequency tuner also allows the cathodes to be tested at a wide range of longitudinal positions inside the cavity, which results in a drastically different field on the cathode surface for the same input rf power [29]. In this experiment, the cathode surface was set to be flush with the rf gun back wall. At this position, $\sim 227 \mathrm{~W}$ of input power is required to obtain a $1 \mathrm{MV} / \mathrm{m}$ cathode electric field. Vacuum in the beam line was maintained below $5 \times 10^{-9}$ Torr.

Diagnostics used in the experiment include a directional coupler to monitor the input and reflected rf power, an $\mathrm{rf}$ pickup installed at the gun side wall to detect the field profile, an aluminum block acting as a Faraday cup at the gun exit to collect the field emission current, and yttriumaluminum-garnet (YAG) screens to observe the beam transverse profile along the beam line. There was also an imaging

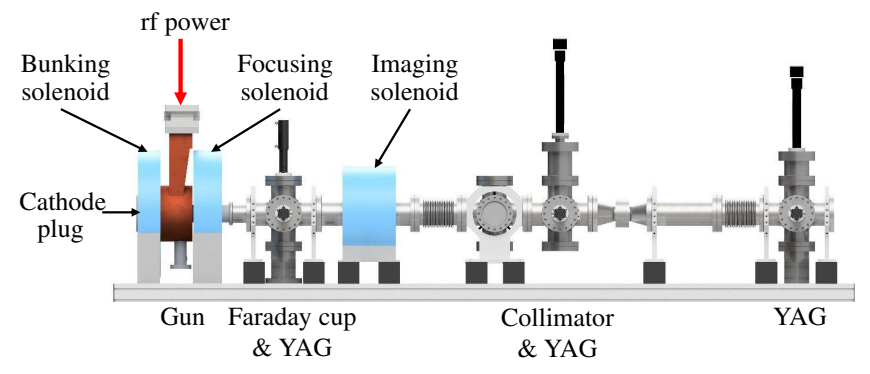

FIG. 4. Layout of the ACT beam line at AWA. 


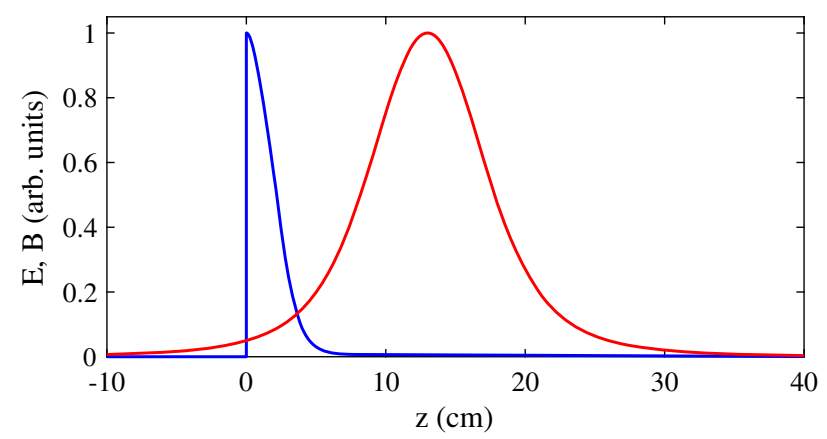

FIG. 5. Longitudinal field profile of the normalized electric field of the rf gun (blue curve) and the normalized magnetic field of the focusing solenoid (red curve). The electric field decreases monotonically from the cathode $(z=0)$ to the gun exit $(z=7.7 \mathrm{~cm})$ due to the single-cell design.

system consisting of solenoids and a collimator to obtain the field emitter distribution with a high resolution [12,30]. A photomultiplier tube with a fluorescent screen sensitive to $\mathrm{x}$ rays was placed near the gun for machine interlock purposes in the event of $\mathrm{rf}$ breakdown [37]. The strength of the focusing solenoid (denoted as $B_{f}$ ) was used to maximize the electron beam capture ratio at the Faraday cup. The longitudinal on-axis field profiles of the rf gun and the focusing solenoid are illustrated in Fig. 5.

\section{HIGH POWER TEST}

\section{A. Field emission charge collection}

The capture ratio of the Faraday cup depends on the rf gun field as well as the focusing solenoid strength. Determining the capture ratio under various circumstances is critical for the interpretation of the field emission properties in the measurement. This subsection introduces the simulation and experimental efforts to ensure effective charge collection as well as to determine the capture ratio.

In this subsection, $\left|E_{c}(t)\right|$ denotes the macroscopic cathode field amplitude within the $6 \mu \mathrm{s}$ rf pulse, $E_{c \text {, max }}$ denotes its maximum value, and $E_{c}(t)=\left|E_{c}(t)\right| \cos (\omega t)$ denotes the transient cathode field where $\omega$ is the operation frequency. Since the rf pulse length is much longer than the rf cycle $(1 / 1.3 \mathrm{GHz}=769.2 \mathrm{ps})$, the slow variation of the field amplitude is ignored and $\left|E_{c}(t)\right|$ is treated as a constant $\left|E_{c}\right|$ in each rf cycle. $\eta_{\text {cycle }}$ and $\eta_{\text {pulse }}$ denote the capture ratio within one rf cycle and one rf pulse, respectively.

In the equations that follow, the units of the electric field, emission current, effective emission area, and work function are $\mathrm{V} / \mathrm{m}, \mathrm{A}, \mathrm{m}^{2}$, and $\mathrm{eV}$, respectively.

\section{Capture ratio within one rf cycle}

According to the Fowler-Nordheim (F-N) equation, the transient field emission current when the cathode field is positive $[\cos (\omega t)>0]$ can be expressed as $[37,38]$

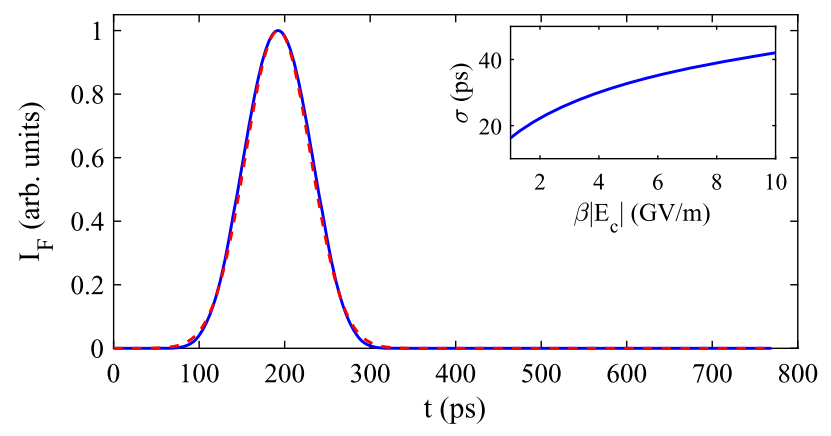

FIG. 6. Emission profile within one rf cycle based on Eq. (1) (blue solid line) and its Gaussian distribution approximation (red dashed line). Inset: The standard deviation of the Gaussian distribution as a function the maximum microscopic cathode field.

$$
\begin{aligned}
I_{F}(t)= & \frac{1.54 \times 10^{-6} \times 10^{4.52 \phi^{-0.5} A_{e}\left[\beta E_{c}(t)\right]^{2}}}{\phi} \\
& \times \exp \left[-\frac{6.53 \times 10^{9} \phi^{1.5}}{\beta E_{c}(t)}\right],
\end{aligned}
$$

where $\phi$ is the work function. The emission profile can be approximated by a Gaussian distribution [39] whose standard deviation $\sigma$ depends on the maximum microscopic cathode field $\beta\left|E_{c}\right|$, as illustrated in Fig. 6 .

As the emission period covers $180^{\circ}$ in the rf cycle, electrons experience different accelerating fields and focusing strengths depending on their emitting phases. A portion of the field emission electrons cannot be captured by the Faraday cup due to the finite gun aperture $(40 \mathrm{~mm}$ in diameter) and the limited Faraday cup size (64 $\mathrm{mm}$ tall and $71 \mathrm{~mm}$ wide), as well as the beam dynamics inside the rf gun.

Beam dynamics simulations with ASTRA [40] have therefore been conducted to examine the capture ratio, in which the electrons were emitted uniformly from the $(\mathrm{N}) \mathrm{UNCD}$ covered area $(18 \mathrm{~mm}$ in diameter) on the cathode with an initial kinetic energy of $0.1 \mathrm{eV}$. The longitudinal emission profile was set to be Gaussian with $\sigma$ determined by the fitted $\beta\left|E_{c}\right|$ (introduced in the following sections). The blue line in Fig. 7 illustrates an example of the simulated capture ratio $\eta_{\text {cycle }}$ dependence on focusing solenoid strength $B_{f}$, with $\left|E_{c}\right|$ of $42 \mathrm{MV} / \mathrm{m}, \beta$ of 179 , and $\beta\left|E_{c}\right|$ of $7.5 \mathrm{GV} / \mathrm{m}$. It shows that over $90 \%$ of the emitted electrons can be captured by the Faraday cup when $B_{f}$ is set to $750 \mathrm{G}$.

\section{Capture ratio within one rf pulse with fixed $E_{c, \max }$}

Within the $6 \mu \mathrm{s}$ rf pulse, the macroscopic cathode electric field amplitude varies due to the finite pulse length and the filling time of the standing-wave cavity, as illustrated in Fig. 8. Therefore, $\eta_{\text {cycle }}$ is also time dependent, and $\eta_{\text {pulse }}$ can be defined as 


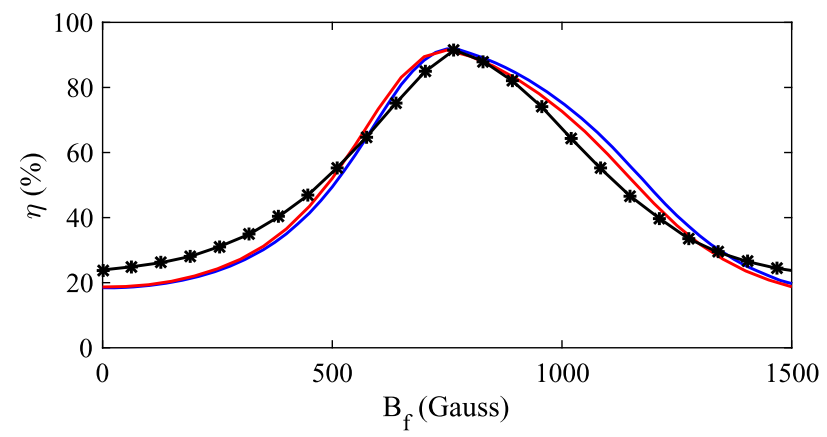

FIG. 7. The capture ratio of the Faraday cup with $\beta$ of 179. Blue line: $\eta_{\text {cycle }}$ with $\left|E_{c}\right|$ of $42 \mathrm{MV} / \mathrm{m}$; red line: $\eta_{\text {pulse }}$ with $E_{c \text {, max }}$ of $42 \mathrm{MV} / \mathrm{m}$; black line: measured charge at $E_{c, \max }$ of $42 \mathrm{MV} / \mathrm{m}$ with the maximum value normalized to the highest capture ratio in the simulation.

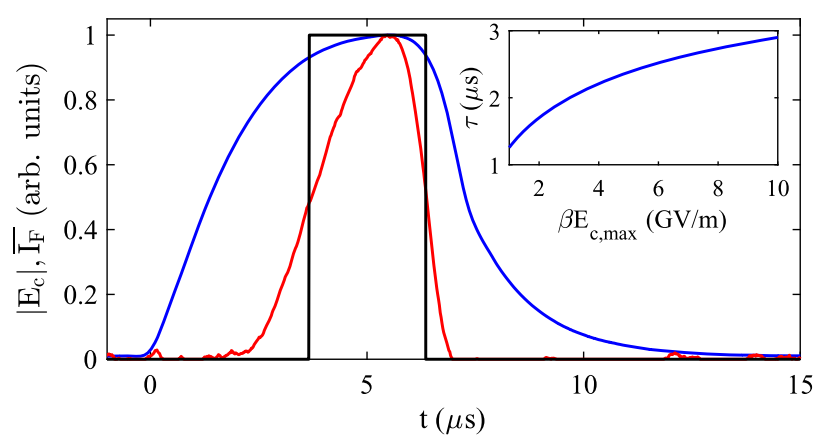

FIG. 8. Blue line: The normalized cathode field amplitude $\left|E_{c}(t)\right|$ measured by the rf pickup. Red line: The predicted average emission current $\overline{I_{F}(t)}$ by Eq. (3). Black line: The square pulse approximation of the emission profile. Inset: The width of the square emission profile as a function of $\beta E_{c, \max }$.

$$
\eta_{\text {pulse }}=\frac{\int \eta_{\text {cycle }}(t) \overline{I_{F}(t)} d t}{\int \overline{I_{F}(t)} d t}
$$

where $\overline{I_{F}(t)}$ denotes the average emission current within one rf cycle that can be expressed as [37]

$$
\begin{aligned}
\overline{I_{F}(t)}= & \frac{5.7 \times 10^{-12} \times 10^{4.52 \phi^{-0.5}} A_{e}\left[\beta\left|E_{c}(t)\right|\right]^{2.5}}{\phi^{1.75}} \\
& \times \exp \left[-\frac{6.53 \times 10^{9} \phi^{1.5}}{\beta\left|E_{c}(t)\right|}\right] .
\end{aligned}
$$

In ASTRA simulations of the capture ratio within one $\mathrm{rf}$ pulse with fixed $E_{c, \text { max }}, \eta_{\text {cycle }}$ at various field levels were individually studied in the same manner as described in the previous subsection; then $\eta_{\text {pulse }}$ was calculated using the predicted emission profile as illustrated in Fig. 8. For each $\left|E_{c}\right|, \beta$ was assumed to be constant and $\sigma$ of the longitudinal emission profile was adjusted based on $\beta\left|E_{c}\right|$. The red line in Fig. 7 illustrates the $\eta_{\text {pulse }}$ dependence on focusing solenoid strength $B_{f}$, with $E_{c \text {,max }}$ of $42 \mathrm{MV} / \mathrm{m}$ and $\beta$ of 179 , which shows good agreement with the experimental results.

In Fig. 7, the blue and the red lines are very similar to each other, which implies that $\eta_{\text {pulse }}$ is dominated by $\eta_{\text {cycle }}$ at $E_{c, \text { max }}$. This can be understood since $\overline{I_{F}(t)}$ is highly sensitive to $\left|E_{c}\right|$. As illustrated in Fig. $8, \overline{I_{F}(t)}$ drops to $1 \%$ of its maximum value when $\left|E_{c}\right|$ decreases by only $20 \%$. The capture ratio study can therefore be simplified by using a square emission profile with an average emission current of $\overline{I_{F, \text { max }}}$ calculated by using $\left|E_{c}\right|=E_{c \text {, max }}$ in Eq. (3), as illustrated in Fig. 8. The width of the square pulse is set as $\tau=\int \overline{I_{F}(t)} d t / \overline{I_{F, \text { max }}}$ so as to keep the same charge. It depends on $\beta E_{c, \max }$, as calculated by Eq. (3) and illustrated in the inset in Fig. 8.

\section{Capture ratio within one rf pulse with various $E_{c, \max }$}

The dependence of $\overline{I_{F, \text { max }}}$ on $E_{c, \text { max }}$ can be plotted in the $1 / E_{c, \text { max }}-\lg \left(\overline{I_{F, \text { max }}} / E_{c, \text { max }}^{2.5}\right)$ coordinate (also known as the F-N coordinate). When space-charge effects do not significantly influence field emission, the dependence of $\lg \left(\overline{I_{F, \max }} / E_{c, \text { max }}^{2.5}\right)$ on $1 / E_{c, \text { max }}$ is linear [41,42], and then $\beta$ and $A_{e}$ can be found from a fit as follows [37]:

$$
\begin{aligned}
\beta & =\frac{-2.84 \times 10^{9} \phi^{1.5}}{s}, \\
A_{e} & =\frac{10^{y_{0}} \phi^{1.75}}{5.7 \times 10^{-12} \times 10^{4.52 \phi^{-0.5}} \beta},
\end{aligned}
$$

where $s$ and $y_{0}$ are the slope and the $y$ axis intercept of the linear dependence, respectively.

Experimental studies of emission properties usually record various electric field levels and the corresponding field emission current in order to fit $\beta$ and $A_{e}$. The capture ratio under these field levels may not be the same, especially in rf structures with complicated geometries, which could lead to inaccurate results. This effect, however, was seldom considered in previous research.

This effect has been carefully simulated in the presented study. As described previously, the emission profile in this simulation step was approximated by a square pulse with its width determined by $\beta E_{c \text {,max }}$. The value of $\beta$ paired with each $E_{c, \max }$ was kept constant with the assumption that the surface condition does not change during the measurement. This assumption is valid, since measurements usually took less than $300 \mathrm{rf}$ pulses ( $2.5 \mathrm{~min})$ without any rf breakdown, compared to the entire experimental period of tens of hours with thousands of rf breakdowns. The variation of $E_{c \text {, max }}$ in the simulation was set to be $20 \%$, close to the one used in the experiment, which was limited by the minimal detectable charge of the Faraday cup. The simulation results, as illustrated in Fig. 9, suggest that a similar capture ratio (with a maximum difference of less than 2\%) can be achieved 

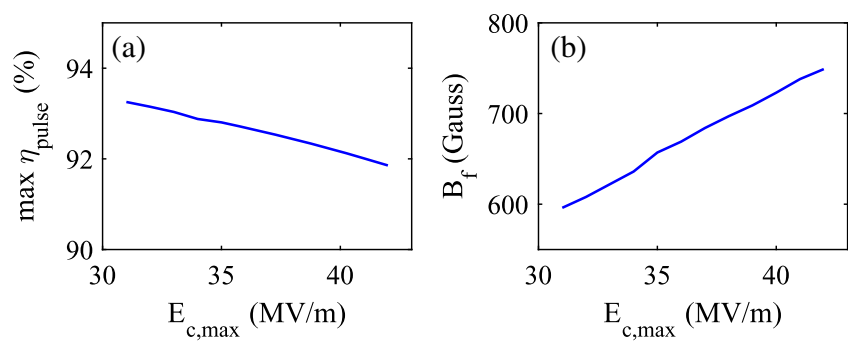

FIG. 9. The maximum $\eta_{\text {pulse }}$ (a) and the corresponding $B_{f}$ (b) as a function of $E_{c, \max }$. In this simulation, $\beta$ is fixed at 179 .

by adjusting $B_{f}$, which supports the accuracy of the fitted results in the following sections.

\section{B. Field emitter distribution}

The field emitter distribution on the (N)UNCD cathode was studied with a low resolution by taking regular YAG images at the gun exit and with a high resolution using the in situ field emission imaging system at the downstream end of the beam line.

\section{Low-resolution observation at the gun exit}

Because of the detachable cathode design, field emission electrons may come from the edge of the cathode or insertion hole rather than the cathode itself. The two sources cannot be distinguished by the Faraday cup. Therefore, a YAG screen (denoted as $\mathrm{YAG}_{1}$ ) located at the same location as the Faraday cup was used to evaluate the emission current from these sources.

ASTRA simulations have been conducted to predict the transverse electron distribution at the $\mathrm{YAG}_{1}$ position. Six $\varnothing 0.2 \mathrm{~mm}$ field emitters were placed on the cathode edge in the simulation, as illustrated in Fig. 10(a). Electrons emit uniformly within each emitter with an initial kinetic energy of $0.1 \mathrm{eV}$. They emit during $180^{\circ}$ of the rf phase when the electric field is positive. The longitudinal profile during one rf cycle has a Gaussian distribution as described in previous sections. The simulated $\mathrm{YAG}_{1}$ image is illustrated in Fig. 10(b). The line-shaped pattern is caused by the wide energy spread of the beam from the broad emission phase, which is common for field emission in rf guns [12,43-46].

The simulation results are compared with the experimental observation under the same $E_{c, \text { max }}$ and $B_{f}$ to identify the source of emission current. With the (N)UNCD cathode, the line-shaped pattern marked by the red circle in Fig. 10(c) has a similar shape and location as that caused by an edge emitter in the ASTRA simulation, which indicates that there was one emitter at the edge of the cathode or insertion hole. The rest of the observed pattern on $\mathrm{YAG}_{1}$ is caused by emitters on the (N)UNCD material, whose total brightness is much higher than that of the edge emitter. Therefore, the collected charge should be dominated by $(\mathrm{N}) \mathrm{UNCD}$ emitters rather than the edge emitter. In comparison, the experimental
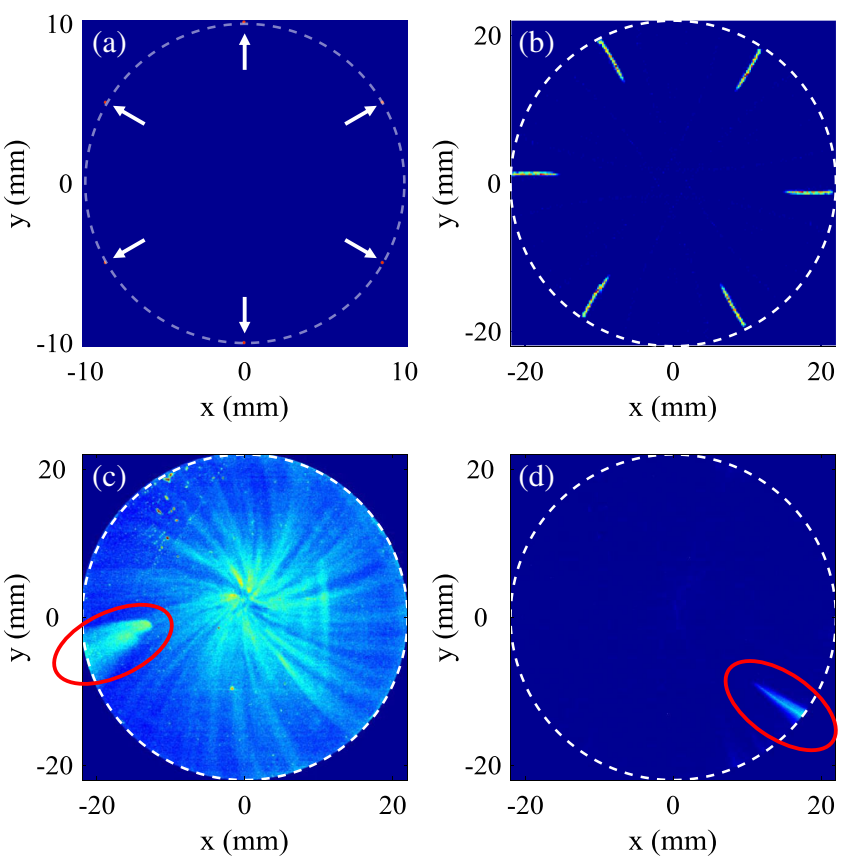

FIG. 10. (a) The initial edge emitters distribution used in the ASTRA simulation. The white dashed circle represents the $\varnothing 20 \mathrm{~mm}$ cathode edge, and the white arrows point to the six emitters. (b) Simulated transverse distribution of field emission electrons from the six emitters on $\mathrm{YAG}_{1}$. (c) Experimental observation with the (N)UNCD cathode on $Y_{1} G_{1}$. (d) Experimental observation with a molybdenum cathode on $\mathrm{YAG}_{1}$. In (b)-(d), the images were simulated or taken with the same $E_{c \text {, max }}$ and $B_{f}$ settings. The white dashed circle represents the $\varnothing 44 \mathrm{~mm}$ YAG boundary. The red circles mark electrons from edge emitters.

result using a molybdenum cathode without (N)UNCD material clearly shows that the emission was mainly from edge emitters, as illustrated in Fig. 10(d).

\section{High-resolution observation at downstream end of the beam line}

The high-resolution field emission imaging system $[12,30]$ was applied to improve the image resolution of the field emitter distribution on the cathode. In this system, a solenoid (the imaging solenoid in Fig. 4, with the strength denoted as $B_{i}$ ) is used to focus the beam. The focal length depends on the beam energy, which is determined by the emitting phase. Electrons with certain energies (emitting phases) can be selected by placing a collimator with a small aperture after the solenoid. The transverse electron distribution on the last YAG screen of the beam line (denoted as $\mathrm{YAG}_{3}$ ) can be used to reproduce the field emitter distribution on the cathode with a certain magnification and rotation. The ASTRA simulation study illustrated in Fig. 11 demonstrates the working principle of the imaging system.

The resolution of the system determines the capability of distinguishing emitters on the cathode. Because of the axial 

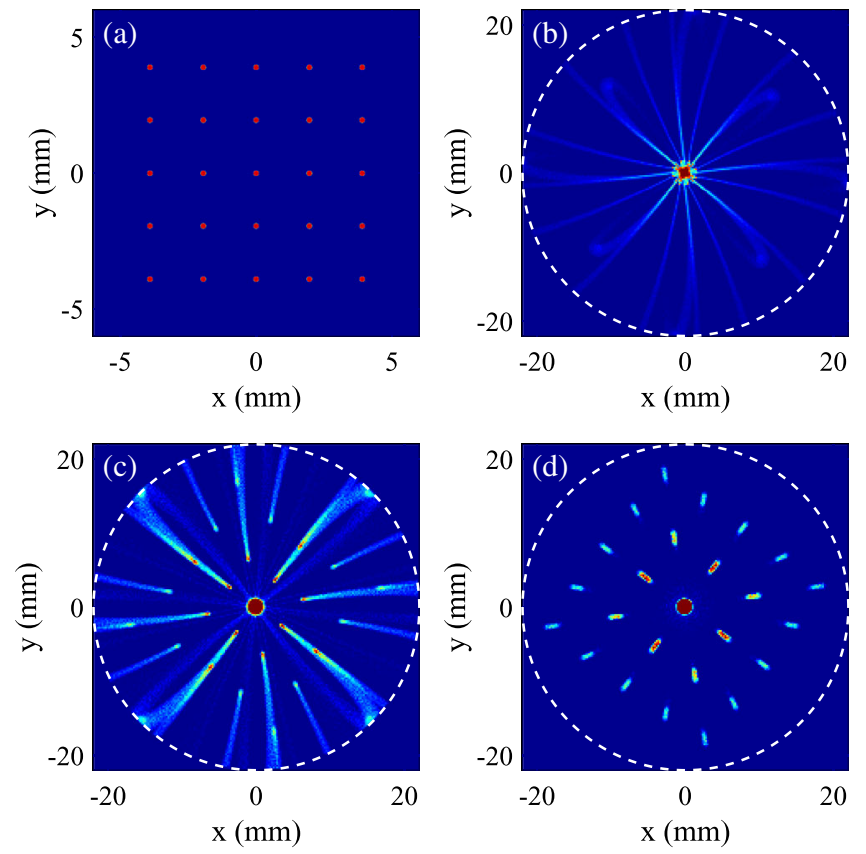

FIG. 11. ASTRA simulation results of the in situ field emission imaging system. (a) Field emitter distribution on the cathode, which consists of $25 \varnothing 0.2 \mathrm{~mm}$ emitters separated by $1.77 \mathrm{~mm}$. (b) Electron transverse distribution at the collimator position. (c) Electron transverse distribution on $\mathrm{YAG}_{3}$ without applying the collimator. (d) Electron transverse distribution on $\mathrm{YAG}_{3}$ with a $\varnothing 1 \mathrm{~mm}$ collimator. In (b)-(d), the white dashed circles represent the $\varnothing 44 \mathrm{~mm}$ YAG boundary.

symmetry of the imaging system, the resolution can be defined in the radial and angular directions (denoted as $R_{\rho}$ and $R_{\varphi}$, respectively). Assuming electrons of a zero-size emitter, after collimation they follow Gaussian distributions in both the radial and angular directions with standard deviations of $\delta_{\rho}$ and $\delta_{\varphi}$. The resolutions of the imaging system can be expressed as $[12,30]$

$$
\begin{aligned}
& R_{\rho}=2.35 \times \frac{\delta_{\rho}}{\overline{\mathrm{mag}}}, \\
& R_{\varphi}=2.35 \times \delta_{\varphi} \rho_{0},
\end{aligned}
$$

where $\overline{\text { mag }}$ is the average magnification and $\rho_{0}$ is the emitter radial position on the cathode.

Under the experimental conditions $\left(E_{c, \max }\right.$ of $36 \mathrm{MV} / \mathrm{m}$, $B_{f}$ of $750 \mathrm{G}, B_{i}$ of $250 \mathrm{G}$, and the aperture diameter of $1 \mathrm{~mm}), R_{\rho}, R_{\varphi}$, and $\overline{\mathrm{mag}}$ are simulated to be $\sim 300 \mu \mathrm{m}$, $\sim 10 \mu \mathrm{m}$, and 3.7, respectively. It should be noted that the resolution is not adequate to resolve the fine structure of (N)UNCD emitters whose sizes were determined to be on the order of microns and below in previous dc studies $[18,27,28]$. The achievable resolution in the experiment could be worse than in the simulation due to the camera resolution, the low signal-to-noise ratio images, the rf amplitude jitter, the variation of $E_{c}$ within the rf pulse, etc.
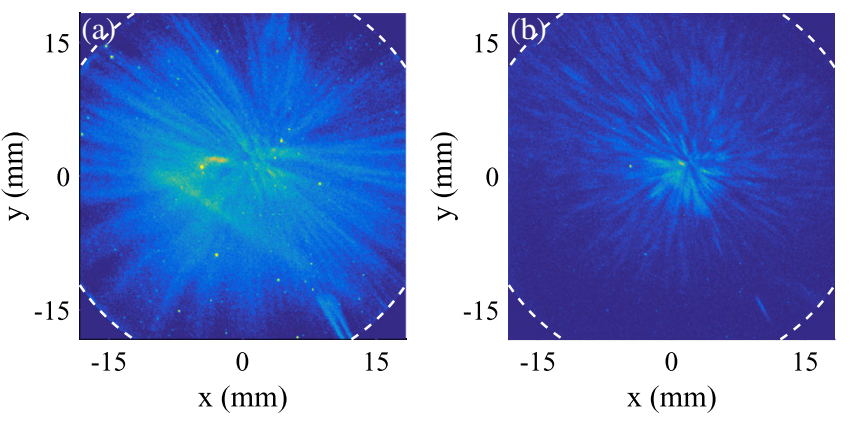

FIG. 12. Field emission images obtained on $\mathrm{YAG}_{3}$ without a collimator (a) and with a $\varnothing 1 \mathrm{~mm}$ aperture (b). The white dashed circles represent the $\varnothing 44 \mathrm{~mm}$ YAG boundary.

As shown in the experiment, however, the in situ imaging system is valuable for distinguishing isolated emitters on the cathode.

Field emission images on $\mathrm{YAG}_{3}$ were taken with the high-resolution in situ field emission imaging system when the cathode field was conditioned to $36 \mathrm{MV} / \mathrm{m}$, as illustrated in Fig. 12. Each short line-shaped pattern after applying the collimator is caused by a single emitter as suggested in the ASTRA simulation. The observation clearly shows that the field emission current was not uniform from the entire $(\mathrm{N}) \mathrm{UNCD}$ cathode but had contributions from a large number of isolated emitters. Given the imaging magnification of 3.7 and YAG diameter of $44 \mathrm{~mm}$, the field of view on the cathode that can be observed on $\mathrm{YAG}_{3}$ is $~ \varnothing 11.9 \mathrm{~mm}$. Over 100 emitters can be found within this range by counting the short lines, which corresponds to an emitter density above $100 / \mathrm{cm}^{2}$. The isolated emitter behavior and the emitter density agree with previous observations in dc setups $[18,27,28]$.

\section{C. rf conditioning}

The field emission properties of (N)UNCD were studied during $\mathrm{rf}$ conditioning by gradually increasing the gun field, $E_{c, \text { max }}$, from $\sim 8$ to $\sim 42 \mathrm{MV} / \mathrm{m}$. The increment of $E_{c, \text { max }}$ was $\sim 0.5 \mathrm{MV} / \mathrm{m}$ in the conditioning process. The breakdown rate could be as high as $1 \times 10^{-1}$ per pulse immediately after the increment. $E_{c, \max }$ was not raised until the breakdown rate decreased to $1 \times 10^{-3}$ per pulse. When a continuous breakdown occurred, the field was reduced to a much lower level until the breakdown stopped, and then the field was pushed back to the breakdown threshold. The entire rf conditioning and measurement process lasted for $40 \mathrm{~h}$.

When conditioned to certain $E_{c \text {,max }}$ levels, e.g., $20 \mathrm{MV} / \mathrm{m}, 40 \mathrm{MV} / \mathrm{m}$, etc., $E_{c, \text { max }}$ was maintained until the rf breakdown rate dropped below $5 \times 10^{-4}$ per pulse. Then $E_{c \text {,max }}$ was gradually decreased and $B_{f}$ was adjusted at each $E_{c, \text { max }}$ to maximize the captured current. According to the aforementioned simulation analysis, the maximum 

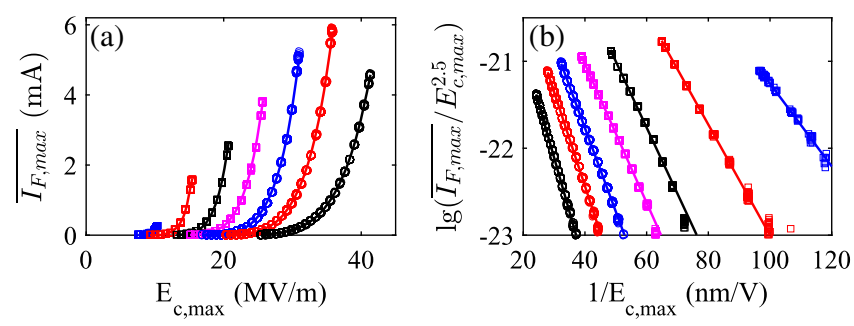

FIG. 13. The dependence of $\overline{I_{F, \max }}$ on $E_{c \text {, max }}$ during rf conditioning, plotted in the $E_{c, \text { max }}-\overline{I_{F, \max }}$ coordinate (a) and the F-N coordinate (b). The dots denote the experimental data, and the lines represent the linear fitting results from the F-N coordinate. The seven curves, from left to right in (a) and from right to left in (b), were taken when $E_{c, \max }$ reached $10,15,21,26,31,36$, and $42 \mathrm{MV} / \mathrm{m}$, respectively.

capture ratio remains nearly unchanged with $E_{c, \max }$, which ensures accuracy when fitting for $\beta$ and $A_{e}$. The dependence of $\overline{I_{F, \text { max }}}$ on $E_{c, \text { max }}$ is illustrated in Fig. 13. The good linearity in the F-N coordinate suggests that the emission was not space-charge limited $[41,42]$. It can be seen that $\overline{I_{F, \text { max }}}$ at a fixed field level kept decreasing during rf conditioning. Meanwhile, the maximum achievable $\overline{I_{F, \max }}$ first rose during the conditioning process, reaching $\sim 6 \mathrm{~mA}$ at $E_{c, \text { max }}=36 \mathrm{MV} / \mathrm{m}$ and then dropping to $\sim 5 \mathrm{~mA}$ at $E_{c, \text { max }}=42 \mathrm{MV} / \mathrm{m}$.

$\beta$ and $A_{e}$ were obtained from fits of the experimental results using Eq. (4) and assuming $\phi=4.0 \mathrm{eV}$. The
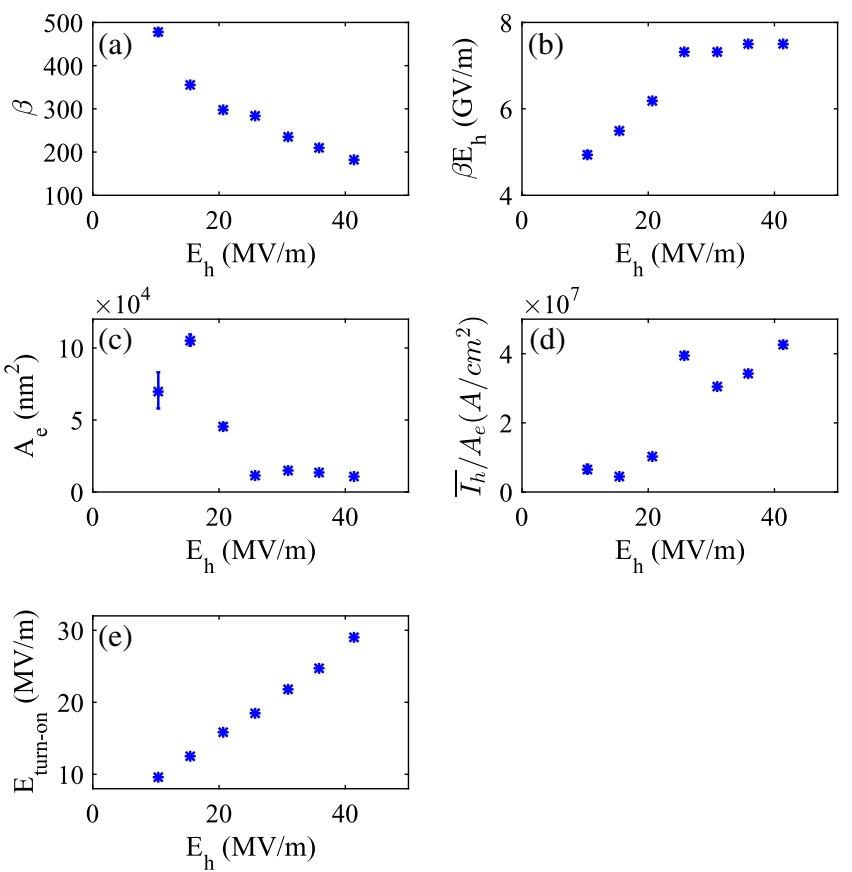

FIG. 14. Evolution of field emission properties during rf conditioning. (a) The field enhancement factor. (b) The maximum microscopic electric field. (c) The effective emission area. (d) The current density. (e) The turn-on field.

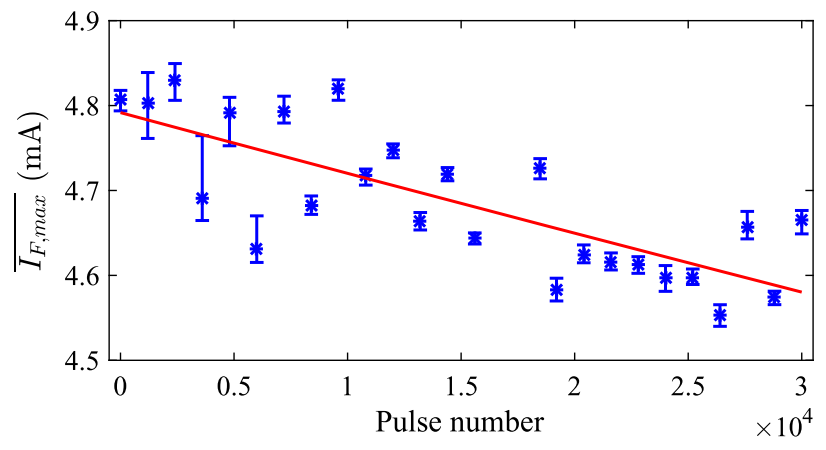

FIG. 15. The current evolution during the 4-h longevity measurement. Blue dots: Measurement results; red line: fitting to $I(t)=I_{0} \exp \left(-t / \tau_{l}\right)$.

microscopic field level, the current density, and the turnon field were derived accordingly, as illustrated in Fig. 14. The turn-on field $E_{\text {turn-on }}$ is defined as the macroscopic field when $\overline{I_{F, \max }}$ reaches $0.1 \mathrm{~mA}$. In Fig. $14, E_{h}$ and $\overline{I_{h}}$ denote the highest achieved $E_{c, \text { max }}$ and the corresponding $\overline{I_{F, \max }}$, respectively. The $\beta$ value kept decreasing while the turn-on field kept increasing during the rf conditioning, while the maximum microscopic field level $\beta E_{h}$ first increased and then reached a stable level of $\sim 7.5 \mathrm{GV} / \mathrm{m}$. This value agrees with the one in a previous study where $E_{h}$ reached $\sim 70 \mathrm{MV} / \mathrm{m}$ [8]. The $A_{e}$ value decreased by one order of magnitude to $1 \times 10^{4} \mathrm{~nm}^{2}$, and the current density reached $\sim 4 \times 10^{7} \mathrm{~A} / \mathrm{cm}^{2}$. The behavior of $\beta$ and the turn-on field are analyzed in the discussion section.

\section{Longevity}

The longevity of the (N)UNCD cathode was tested when $E_{c, \max }$ reached $42 \mathrm{MV} / \mathrm{m}$. In the 4-h measurement, $\sim 3 \times 10^{4} \mathrm{rf}$ pulses or $\sim 1 \times 10^{8} \mathrm{rf}$ cycles were accumulated and the current dropped by only $\sim 4 \%$, as illustrated in Fig. 15. The number of rf pulses was based on the $2.5 \mu \mathrm{s}$ square emission pulse and $1.3 \mathrm{GHz}$ frequency. The current is assumed to follow $I(t)=I_{0} \exp \left(-t / \tau_{l}\right)$, where $\tau_{l}$ denotes the lifetime of the cathode after which the current will be
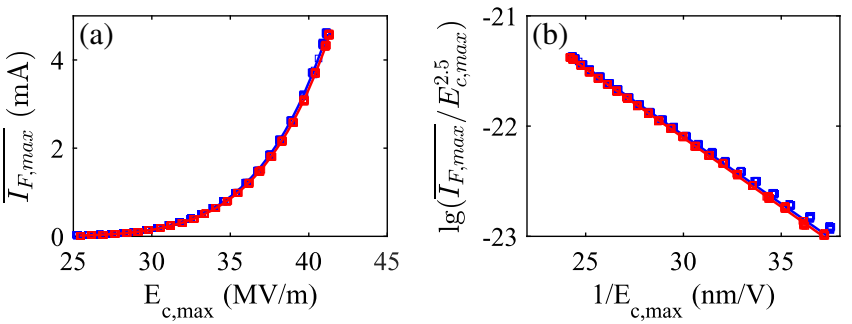

FIG. 16. The dependence of $\overline{I_{F, \text { max }}}$ on $E_{c, \text { max }}$ before (blue) and after (red) the longevity measurement, plotted in the $E_{c \text {, max }}-$ $\overline{I_{F, \max }}$ coordinate (a) and the F-N coordinate (b). The dots denote the experimental data, and the lines represent the linear fitting results from the F-N coordinate. 
$37 \%$ of its initial value. From the 4-h measurement data, $\tau_{l}$ can be fitted to be $\sim 92 \mathrm{~h}$.

Only one breakdown occurred during the measurement. The good longevity and low rf breakdown rate $\left(3 \times 10^{-5}\right.$ per pulse) demonstrate the promising potential of $(\mathrm{N})$ UNCD material in rf injector applications.

The field emission properties were determined before and after the 4-h longevity measurement, and the difference was negligible, as illustrated in Fig. 16. Together with the significant variation during $\mathrm{rf}$ conditioning, it suggests that the evolution of the field emission properties was mainly caused by rf breakdowns rather than accumulated rf pulses.

\section{DISCUSSION}

In this section, the band diagram of the (N)UNCD material is used to interpret the experimental phenomena observed in this study. These include the nonuniform graphite-to-diamond content distribution revealed by the Raman spectra measurement, nonuniform field emitter distribution revealed by the in situ imaging system, the threefold decrease of $\beta$ and threefold increase of $E_{\text {turn-on }}$ revealed during rf conditioning, and the different behaviors of the surface during the conditioning process when there were remarkable changes of emission properties compared to the longevity measurement when the emission properties remained nearly unchanged.

The basic band diagram of (N)UNCD, viz. the density of states as a function of the energy is illustrated in Fig. 17(a).
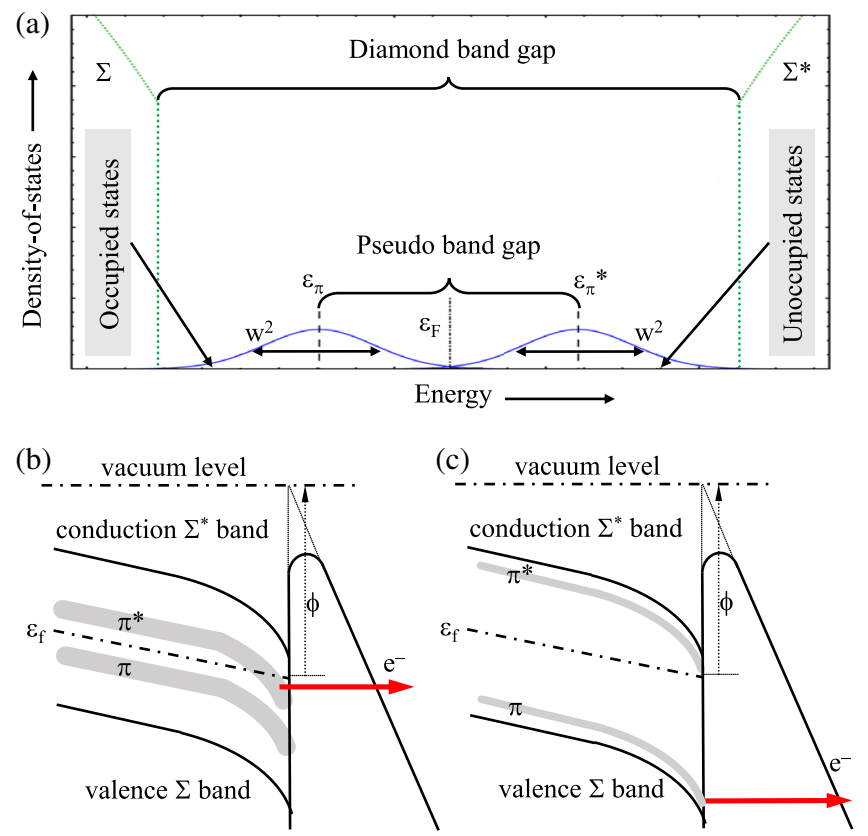

FIG. 17. (a) Density-of-states diagram of (N)UNCD. Surface barrier under the same external macroscopic electric field for $s p^{2}$-rich sites (b) and $s p^{2}$-poor sites (c). The complete selfconsistently solved theory based on Stratton-Baskin-Lvov-Fursey formalism can be found in Ref. [26].
It is modeled as a combination of $s p^{2}$ (i.e., graphitic grain boundary) induced $\pi$ bands inserted into the fundamental band gap of diamond (i.e., host matrix of diamond grains which are $s p^{3}$-bonded carbon). The states in the $\pi-\pi^{*}$ bands can be approximated by Gaussian functions centered at $\varepsilon_{\pi}$ and $\varepsilon_{\pi^{*}}$ with variance $w^{2}$. It is assumed that $\pi-\pi^{*}$ and $\Sigma-\Sigma^{*}$ bands are mirrored with respect to the Fermi level that is pinned inside the pseudo band gap formed by the $\pi-\pi^{*}$ states. In synthesis, the addition of nitrogen to UNCD always adds a more graphitic phase $[47,48]$, more states in the $\pi-\pi^{*}$ bands with an increased intensity and width, and a smaller distance between $\varepsilon_{\pi}$ and $\varepsilon_{\pi^{*}}$. The general trend to expect then is that the overlap between $\pi-\pi^{*}$ bands increases. Consequently, the pseudo band gap becomes smaller (optically darker films [47]) which yields highly conductive semimetallic (N)UNCD.

(N)UNCD is compositionally nonuniform (as illustrated in Fig. 3), and two extreme cases are considered for simplicity: $s p^{2}$-rich (N)UNCD sites in Fig. 17(b) and $s p^{2}$-poor (N)UNCD sites in Fig. 17(c). The partial penetration of the electric field into the material leads to band bending near the surface. In $s p^{2}$-rich (N)UNCD, the $\pi^{*}$ band can be therefore lower than the Fermi level in the nearsurface layer. Given the strong overlap (enhanced charge exchange) between $\pi-\pi^{*}$ bands, a large number of electrons are expected to collect near the surface (metallizing it). These electrons tunnel through the surface barrier with a larger probability, as they have higher energy positions inside the material as compared to the states normally occupied without an external field perturbation. The result is a high charge carrier concentration and metalliclike behavior which further leads to a high field enhancement factor and low turn-on field [26]. In $s p^{2}$-poor (N)UNCD, on the other hand, electrons could come only from the $\pi$ (or $\Sigma$ ) band with a lower probability of tunneling through the thicker surface barrier. The result is a low field enhancement factor and high turn-on field [26]. Under the same macroscopic field, the current density of $s p^{2}$-rich $(\mathrm{N})$ UNCD should be higher than that of $s p^{2}$-poor (N)UNCD. This speculation is consistent with the nonuniform emitter distribution revealed by the in situ field emission images.

Therefore, the mechanism of (N)UNCD field emission during rf conditioning can be interpreted as follows. (i) Starting at a low surface field, the emission current is dominated by $s p^{2}$-rich (N)UNCD sites which causes the high $\beta$ and low $E_{\text {turn-on }}$ observed in the measurement. (ii) When the surface field is increased during the conditioning progress, a high localized emission current leads to thermally driven degradation and extinction of originally strong emitters [49] which may be presented as rf breakdowns. (iii) At higher fields, $s p^{2}$-poor $(\mathrm{N}) \mathrm{UNCD}$ sites dominate the emission as $s p^{2}$-rich locations on the (N)UNCD surface are consumed as conditioning proceeds, leading to low $\beta$ and high $E_{\text {turn-on. This speculation is }}$ consistent with the monotonically decreasing $\beta$ and the 
increasing $E_{\text {turn-on }}$ observed in the experiment. It is also supported by the observation of dramatic emission property changes during the conditioning process with a large number of rf breakdowns versus nearly unchanged emission properties in the longevity measurement with only one rf breakdown detected.

It should be noted that, in the context of the obtained results and the proposed emission mechanism, the nearsurface region always has a certain amount of accumulated charge that makes the charge-field relation FowlerNordheim-like, with no observable saturation effects [26] due to the low duty cycle of the ACT beam line. Therefore, the application of the classical Fowler-Nordheim law is possible: It simplifies the analysis and makes the comparison between different geometries and material modifications reasonably good. Strictly speaking, the Stratton-BaskinLvov-Fursey formalism should be considered for semiconductor and semimetal material analysis and modeling: This is, however, an exceptionally complex problem that is out of the scope of the presented study.

\section{SUMMARY AND FUTURE STUDIES}

This study systematically benchmarked the field emission properties of a planar nitrogen-incorporated ultrananocrystalline diamond during the $\mathrm{rf}$ conditioning process where the macroscopic field was pushed from $\sim 8$ to $\sim 42 \mathrm{MV} / \mathrm{m}$. The cathode reached a maximum charge of $15 \mathrm{nC}$ and an average emission current of $6 \mathrm{~mA}$ during a $2.5 \mu$ s emission period. The charge dropped by only $\sim 4 \%$ during a 4-h longevity measurement at $42 \mathrm{MV} / \mathrm{m}$ which accumulated $\sim 3 \times 10^{4} \mathrm{rf}$ pulses or $\sim 1 \times 10^{8} \mathrm{rf}$ cycles. The high-resolution field emission images revealed a large number of field emitters on the cathode with a density over $100 / \mathrm{cm}^{2}$. This study demonstrates the good potential of (N)UNCD cathodes for application in FEC-based injectors. The observed conditioning effects can all be reasonably explained using the band structure of the material, which, in turn, provides insights for guiding further material engineering with the goal of improved cathode performance.

Future work includes experiments to study the emittance of (N)UNCD field emission cathodes, a comparison between the trend of the emission area calculated by the F-N equation and that of $s p^{2}$-rich and $s p^{2}$-poor sites directly observed by high-resolution Raman mapping, and the design of electron sources based on the parameter space reported in this manuscript.

\section{ACKNOWLEDGMENTS}

The work at AWA is funded through the U.S. Department of Energy Office of Science under Contract No. DE-AC0206CH11357. The work by M.S. is supported by the U.S. Department of Energy, Office of Science, High Energy Physics under Cooperative Agreement Grant No. DESC0018362 and Michigan State University. The work by
G. C. is supported by National Science Foundation Grants No. NSF-1739150, No. NSF-1535676, and No. DESC0015479. The work by S. V. B. and T. N. is funded from the College of Engineering, Michigan State University, under the Global Impact Initiative. Use of the Center for Nanoscale Materials, an Office of Science user facility, was supported by the U.S. Department of Energy, Office of Science, Office of Basic Energy Sciences, under Contract No. DE-AC0206CH11357. We thank Dr. Evgenya Simakov at Los Alamos National Laboratory for providing the molybdenum cathode.

[1] C. A. Brau, High-brightness electron beams-small freeelectron lasers, Nucl. Instrum. Methods Phys. Res., Sect. A 407, 1 (1998).

[2] G. N. Fursey, Field Emission in Vacuum Microelectronics (Springer Science, New York, 2007).

[3] S. Leemann, A. Streun, and A. Wrulich, Beam characterization for the field-emitter-array cathode-based low-emittance gun, Phys. Rev. Accel. Beams 10, 071302 (2007).

[4] J. Lewellen and J. Noonan, Field-emission cathode gating for rf electron guns, Phys. Rev. Accel. Beams 8, 033502 (2005).

[5] R. Ganter, R. Bakker, C. Gough, M. Paraliev, M. Pedrozzi, F. Le Pimpec, L. Rivkin, and A. Wrulich, Nanosecond field emitted and photo-field emitted current pulses from $\mathrm{ZrC}$ tips, Nucl. Instrum. Methods Phys. Res., Sect. A 565, 423 (2006).

[6] X. Li, M. Li, L. Dan, Y. Liu, and C. Tang, Cold cathode rf guns based study on field emission, Phys. Rev. Accel. Beams 16, 123401 (2013).

[7] P. Piot, C. Brau, B. Choi, B. Blomberg, W. Gabella, B. Ivanov, J. Jarvis, M. Mendenhall, D. Mihalcea, H. Panuganti et al., Operation of an ungated diamond fieldemission array cathode in a L-band radiofrequency electron source, Appl. Phys. Lett. 104, 263504 (2014).

[8] S. V. Baryshev, S. Antipov, J. Shao, C. Jing, K. J. Pérez Quintero, J. Qiu, W. Liu, W. Gai, A. D. Kanareykin, and A. V. Sumant, Planar ultrananocrystalline diamond field emitter in accelerator radio frequency electron injector: Performance metrics, Appl. Phys. Lett. 105, 203505 (2014).

[9] D. Mihalcea, L. Faillace, J. Hartzell, H. Panuganti, S. Boucher, A. Murokh, P. Piot, and J. Thangaraj, Measurement of Ampère-class pulsed electron beams via field emission from carbon-nanotube cathodes in a radiofrequency gun, Appl. Phys. Lett. 107, 033502 (2015).

[10] J. Qiu, S. S. Baturin, K. K. Kovi, O. Chubenko, G. Chen, R. Konecny, S. Antipov, A. V. Sumant, C. Jing, and S. V. Baryshev, Nanodiamond thin film field emitter cartridge for miniature high-gradient radio frequency $\mathrm{X}$-band electron injector, IEEE Trans. Electron Devices 65, 1132 (2018).

[11] X. Li, M. Li, L. Dan, T. He, Y. Liu, Z. Xu, and C. Tang, A double-frequency rf gun for field emission, Nucl. Instrum. Methods Phys. Res., Sect. A 783, 1 (2015).

[12] J. Shao, J. Shi, S. P. Antipov, S. V. Baryshev, H. Chen, M. Conde, W. Gai, G. Ha, C. Jing, F. Wang et al., In Situ Observation of Dark Current Emission in a High Gradient rf Photocathode Gun, Phys. Rev. Lett. 117, 084801 (2016). 
[13] K. B. Teo, E. Minoux, L. Hudanski, F. Peauger, J.-P. Schnell, L. Gangloff, P. Legagneux, D. Dieumegard, G. A. Amaratunga, and W. I. Milne, Carbon nanotubes as cold cathodes, Nature (London) 437, 968 (2005).

[14] Q. Wang, X. Li, Y. Di, C. Yu, X. Zhang, M. Li, and W. Lei, High brightness field emission from printed carbon nanotubes in an S-band microwave gun, J. Appl. Phys. 119, 084504 (2016).

[15] D. Zhou, A. Krauss, L. Qin, T. McCauley, D. Gruen, T. Corrigan, R. P. Chang, and H. Gnaser, Synthesis and electron field emission of nanocrystalline diamond thin films grown from $N_{2} / \mathrm{CH}_{4}$ microwave plasmas, J. Appl. Phys. 82, 4546 (1997).

[16] T. Ikeda and K. Teii, Origin of low threshold field emission from nitrogen-incorporated nanocrystalline diamond films, Appl. Phys. Lett. 94, 143102 (2009).

[17] R. J. Nemanich, J. A. Carlisle, A. Hirata, and K. Haenen, CVD diamond-Research, applications, and challenges, MRS Bull. 39, 490 (2014).

[18] O. Chubenko, S. S. Baturin, K. K. Kovi, A. V. Sumant, and S. V. Baryshev, Locally resolved electron emission area and unified view of field emission from ultrananocrystalline diamond films, ACS Appl. Mater. Interfaces 9, 33229 (2017).

[19] N. Shang, P. Papakonstantinou, P. Wang, A. Zakharov, U. Palnitkar, I.-N. Lin, M. Chu, and A. Stamboulis, Selfassembled growth, microstructure, and field-emission high-performance of ultrathin diamond nanorods, ACS Nano 3, 1032 (2009).

[20] V. Krivchenko, A. Pilevsky, A. Rakhimov, B. Seleznev, N. Suetin, M. Timofeyev, A. Bespalov, and O. Golikova, Nanocrystalline graphite: Promising material for high current field emission cathodes, J. Appl. Phys. 107, 014315 (2010).

[21] K. Teii and M. Nakashima, Synthesis and field emission properties of nanocrystalline diamond/carbon nanowall composite films, Appl. Phys. Lett. 96, 023112 (2010).

[22] I. Levchenko, S. Xu, G. Teel, D. Mariotti, M. Walker, and M. Keidar, Recent progress and perspectives of space electric propulsion systems based on smart nanomaterials, Nat. Commun. 9, 879 (2018).

[23] V. Chatterjee, R. Harniman, P. W. May, and P. Barhai, Direct observation of electron emission from the grain boundaries of chemical vapour deposition diamond films by tunneling atomic force microscopy, Appl. Phys. Lett. 104, 171907 (2014).

[24] R. L. Harniman, O. J. Fox, W. Janssen, S. Drijkoningen, K. Haenen, and P. W. May, Direct observation of electron emission from grain boundaries in CVD diamond by PeakForce-controlled tunnelling atomic force microscopy, Carbon 94, 386 (2015).

[25] E. Wang at Brookhaven National Laboratory (private communication).

[26] O. Chubenko, S. S. Baturin, and S. V. Baryshev, Theoretical evaluation of electronic density-of-states and transport effects on field emission from n-type ultrananocrystalline diamond films, J. Appl. Phys. 125, 205303 (2019).

[27] J. Cui and J. Robertson, Field emission from chemical vapor deposition diamond surface with graphitic patches, J. Vac. Sci. Technol. B 20, 238 (2002).
[28] F. Köck, J. Garguilo, and R. Nemanich, Direct correlation of surface morphology with electron emission sites for intrinsic nanocrystalline diamond films, Diam. Relat. Mater. 13, 1022 (2004).

[29] J. Shao, S. P. Antipov, S. V. Baryshev, H. Chen, M. Conde, D. S. Doran, W. Gai, C. Jing, W. Liu, J. Power et al., Observation of Field-Emission Dependence on Stored Energy, Phys. Rev. Lett. 115, 264802 (2015).

[30] J. Shao, Investigations on rf Breakdown Phenomenon in High Gradient Accelerating Structures (Springer, New York, 2018).

[31] D. Xiang, W.-H. Huang, Y.-C. Du, L.-X. Yan, R.-K. Li, C.-X. Tang, Y.-Z. Lin, S.-J. Park, and J. Park, in Proceedings of the 22nd Particle Accelerator Conference, PAC-2007, Albuquerque, NM (IEEE, New York, 2007), pp. 1049-1051.

[32] H. J. Qian, C. Li, Y. C. Du, L. X. Yan, J. F. Hua, W. H. Huang, and C.X. Tang, Experimental investigation of thermal emittance components of copper photocathode, Phys. Rev. Accel. Beams 15, 040102 (2012).

[33] T. Corrigan, D. Gruen, A. Krauss, P. Zapol, and R. P. Chang, The effect of nitrogen addition to $\mathrm{Ar} / \mathrm{CH}_{4}$ plasmas on the growth, morphology and field emission of ultrananocrystalline diamond, Diam. Relat. Mater. 11, 43 (2002).

[34] T. Nikhar, R. Rechenberg, M. Becker, and S. V. Baryshev, Revisiting Effects of Nitrogen Incorporation and Graphitization on Conductivity of Ultra-nano-crystalline Diamond Films, arXiv:1910.09595.

[35] K. J. Pérez Quintero, S. Antipov, A. V. Sumant, C. Jing, and S. V. Baryshev, High quantum efficiency ultrananocrystalline diamond photocathode for photoinjector applications, Appl. Phys. Lett. 105, 123103 (2014).

[36] G. Chen, G. Adhikari, L. Spentzouris, K. K. Kovi, S. Antipov, C. Jing, W. Andreas Schroeder, and S. V. Baryshev, Mean transverse energy of ultrananocrystalline diamond photocathode, Appl. Phys. Lett. 114, 093103 (2019).

[37] J. Wang and G. Loew, Field emission and rf breakdown in high-gradient room-temperature linac structures, Stanford Linear Accelerator Center, Technical Report No. SLACPUB-7684, 1997.

[38] R. H. Fowler and L. Nordheim, Electron emission in intense electric fields, Proc. R. Soc. A 119, 173 (1928).

[39] R. Huang, D. Filippetto, C. Papadopoulos, H. Qian, F. Sannibale, and M. Zolotorev, Dark current studies on a normal-conducting high-brightness very-high-frequency electron gun operating in continuous wave mode, Phys. Rev. Accel. Beams 18, 013401 (2015).

[40] K. Flöttmann, ASTRA-A space charge tracking algorithm, DESY.

[41] J. Barbour, W. Dolan, J. Trolan, E. Martin, and W. Dyke, Space-charge effects in field emission, Phys. Rev. 92, 45 (1953).

[42] A. Rokhlenko, K. L. Jensen, and J. L. Lebowitz, Space charge effects in field emission: One dimensional theory, J. Appl. Phys. 107, 014904 (2010).

[43] J. Han, J. Bähr, H. Grabosch, M. Krasilnikov, V. Miltchev, A. Oppelt, and B. Petrosyan, in Proceedings of the 21st 
Particle Accelerator Conference, Knoxville, TN, 2005 (IEEE, Piscataway, NJ, 2005), pp. 895-897.

[44] A. Moretti, Z. Qian, J. Norem, Y. Torun, D. Li, and M. Zisman, Effects of high solenoidal magnetic fields on $\mathrm{rf}$ accelerating cavities, Phys. Rev. Accel. Beams 8, 072001 (2005).

[45] D. Dowell, E. Jongewaard, C. Limborg-Deprey, J. Schmerge, and A. Vlieks, in Proceedings of the 22nd Particle Accelerator Conference, PAC-2007, Albuquerque, NM (IEEE, New York, 2007), pp. 1299-1301.

[46] R. Xiang, A. Arnold, T. Kamps, P. Lu, P. Michel, P. Murcek, H. Vennekate, G. Staats, and J. Teichert, Experimental studies of dark current in a superconducting rf photoinjector, Phys. Rev. ST Accel. Beams 17, 043401 (2014).

[47] M. Nesládek, K. Meykens, L. Stals, M. Vaněček, and J. Rosa, Origin of characteristic subgap optical absorption in CVD diamond films, Phys. Rev. B 54, 5552 (1996).

[48] P. Zapol, M. Sternberg, L. A. Curtiss, T. Frauenheim, and D. M. Gruen, Tight-binding molecular-dynamics simulation of impurities in ultrananocrystalline diamond grain boundaries, Phys. Rev. B 65, 045403 (2001).

[49] S. S. Baturin, T. Nikhar, and S. V. Baryshev, Field electron emission induced glow discharge in a nanodiamond vacuum diode, J. Phys. D 52, 325301 (2019). 